\title{
How Nested and Monogamous Infection Networks in Host-Phage Communities Come To Be
}

\author{
Daniel A. Korytowski · Hal L. Smith
}

Received: date / Accepted: date

\begin{abstract}
We show that a chemostat community of bacteria and bacteriophage in which bacteria compete for a single nutrient and for which the bipartite infection network is perfectly nested is permanent, a.k.a. uniformly persistent, provided that bacteria that are superior competitors for nutrient devote the least effort to defence against infection and the virus that are the most efficient at infecting host have the smallest host range. This confirms earlier work of Jover et al [7] who raised the issue of whether nested infection networks are permanent. In addition, we provide sufficient conditions that a bacteria-phage community of arbitrary size with nested infection network can arise through a succession of permanent subcommunties each with a nested infection network by the successive addition of one new population. The same permanence results hold for the monogamous infection network considered by Thingstad [15] but without the tradeoffs.
\end{abstract}

Keywords bacteriophage $\cdot$ competitive exclusion principle $\cdot$ ecological succession $\cdot$ nested infection network $\cdot$ permanence $\cdot$ persistence $\cdot$ predatormediated coexistence

\section{Introduction}

This work is inspired by the recent paper of Jover, Cortez, and Weitz [7]. Noting that empirical studies strongly suggest that the bipartite infection networks observed in bacteria and virus communities tend to have a nested structure characterized by a hierarchy among both host and virus strains which

Daniel A. Korytowski · Hal L. Smith

School of Mathematical and Statistical Sciences

Arizona State University

Tempe, AZ, 85287

E-mail: Daniel.Korytowski@asu.edu

E-mail: halsmith@asu.edu 
constrains which virus may infect which host, they identify key tradeoffs between competitive ability of the bacteria hosts and defence against infection and, on the part of virus, between virulence and transmissibility versus host range such that a nested infection network can be maintained. They find that "bacterial growth rate should decrease with increasing defence against infection" and that "the efficiency of viral infection should decrease with host range". Their mathematical analysis of a Lotka-Volterra model incorporating the above mentioned tradeoffs strongly suggests that the perfectly nested community structure of $n$-host bacteria and $n$-virus is permanent, sometimes also called persistent, or uniformly persistent $[6,11,14]$. Indeed, they establish several necessary conditions for permanence: (1) a positive equilibrium for the system with all host and virus populations at positive density exists, and (2) every boundary equilibrium of the $2 n$-dimensional ordinary differential equations, where one or more population from the nested structure is missing, is unstable to invasion by at least one of the missing populations. They also note that while equilibrium dynamics are rare for such systems, invasability of boundary equilibria can imply invasability of general boundary dynamics provided permanence holds according to results of Hofbauer and Sigmund [6]. However, permanence of a perfectly nested infection network is not established in [7]. The famous example of three-species competition described by May and Leonard [9] shows that the necessary conditions mentioned above are not sufficient for permanence.

Permanence of bacteriophage and bacteria in a chemostat has been established for mathematical models of very simple communities consisting of a single virus and one or two host bacteria in $[12,4]$.

A nested infection network of three bacterial strains and three virus strains has the structure described in the infection Table 1. An ' $x$ ' in the matrix means that the host below is infected by the virus on the left while a blank entry indicates no infection; for example, the second column of three x's indicates that bacteria $H_{1}$ is infected by virus $V_{1}, V_{2}$ and $V_{3}$. Host $H_{1}$ is the least resistant to infection while $H_{3}$ is the most resistant; virus $V_{1}$ specializes on a single host while $V_{3}$ is a generalist, infecting all host.

Table 1 Nested Infection Table for bacteria $H_{i}$ and virus $V_{j}$

\begin{tabular}{|c|c|c|c|}
\hline$V_{3}$ & $\mathrm{x}$ & $\mathrm{x}$ & $\mathrm{x}$ \\
\hline$V_{2}$ & $\mathrm{x}$ & $\mathrm{x}$ & \\
\hline$V_{1}$ & $\mathrm{x}$ & & \\
\hline & $H_{1}$ & $H_{2}$ & $H_{3}$ \\
\hline
\end{tabular}

A community of bacterial populations and virus populations with infection network as in Table 1 could evolve by the sequential addition of one new population starting from a single susceptible host and virus population. Following a mutation or colonization event, a new bacterial population, resistant to the virus, may appear. Subsequently, a mutational event may result in a new virus population capable of infecting both bacterial populations. Clearly, alternat- 
ing the addition of a new bacterial population resistant to all existing virus populations with the addition of a new virus population capable of infecting all pre-existing bacterial populations leads to the community described above.

Just such a sequence of mutational or selection events is observed in chemostat experiments starting from a single bacteria population and a single virus population and leading to a nested infection network. Chao et al [2] describe such a scenario in their experimental observations of E. Coli and phage T7. A bacterial mutant resistant to the virus is observed to evolve first. Subsequently, a viral mutant evolves which is able to infect both bacterial populations. In one replicate, a second bacterial mutant arises which is resistant to both viruses. Pairwise competition experiments between the phage-sensitive bacterial strain and the resistant mutant strains in a virus-free chemostat showed that the resistant mutants were inferior competitors relative to the susceptible strain.

Similar evolutionary scenarios are noted in the review of Bohannan and Lenski [1]. They note that bacterial resistance is often the result of a mutation that affects a receptor used both for bacterial metabolism and as a target for viral adsorption. The modified receptor confers resistance to virus adsorption at the cost of inferior affinity for a resource. Bohannan and Lenski stress that the persistence of a resistant bacterial mutant does not cause extinction of the sensitive bacteria and phage provided that (i) the resistant mutant has reduced competitive ability relative to the sensitive bacteria, and (ii) resistance to the virus is absolute.

Thingstad [15] considered an infection network that we call monogamous where each virus specializes on a single host in his study of mechanisms that control the abundance of bacteria and virus in aquatic systems. See the infection Table 2. A monogamous network might arise simply as an assembly of distantly related or unrelated bacteria populations each with its own specialist virus parasite.

Table 2 Monogamous Infection Table

\begin{tabular}{|c|c|c|c|}
\hline$V_{3}$ & & & $\mathrm{x}$ \\
\hline$V_{2}$ & & $\mathrm{x}$ & \\
\hline$V_{1}$ & $\mathrm{x}$ & & \\
\hline & $H_{1}$ & $H_{2}$ & $H_{3}$ \\
\hline
\end{tabular}

Our goal in this paper is to show that a nested infection network consisting of $n$ bacterial host and $n$ lytic virus is permanent given the trade-offs identified in [7]. Recall that permanence means that there is a positive threshold, independent of positive initial conditions of all populations, which every bacteria and virus density ultimately exceeds. We also show that permanence holds for the monogamous network without trade-offs.

However, we replace the Lotka-Volterra model used by Jover et al [7] by a chemostat-based model where bacterial populations compete for nutrient and virus populations compete for hosts as in $[2,6,12,17,15]$, although we ignore latency of virus infection. Aside from the additional realism of including 
competition for nutrient, our model avoids the non-generic bacterial dynamics of the Lotka-Volterra model which possesses an $n$-1-dimensional simplex of virus-free equilibria.

Chemostat-based models of microbial competition for a single nutrient are known to induce a ranking of competitive ability among the microbes determined by their break-even nutrient concentrations for growth, here denoted by $\lambda$ but often by $R^{*}$ in the ecological literature. The competitive exclusion principle applies: a single microbial population, the one with smallest $\lambda$, drives all others to extinction $[16,13]$ in the absence of virus. In our model of a nested infection network, this host can be infected by every virus strain and as the $\lambda$ value of host strains increases (i.e., it becomes less competitive for nutrient) it is subject to infection by fewer virus strains. Virus populations are ranked by their efficiency at infecting host. The most infection-efficient strain specializes on the host with smallest $\lambda$ and as infection efficiency decreases host range increases so that the virus strain of rank $k$ infects the $k$ most competitive host strains.

Our permanence result is a dramatic example of predator-mediated coexistence. In the absence of phage, only a single bacterial strain can survive. However, the addition of an equal number of phage to our microbial community where the infection network is either monogamous or nested and if the latter, with the trade-offs noted above, leads to the coexistence of all populations. Predator-mediated coexistence was also noted by Thingstad [15] for a Lotka-Volterra model with monogamous infection network.

In fact, we will show that the $n$-bacteria, $n$-virus community can arise through a succession of permanent sub-communities starting with an ancestral community of one susceptible bacterial host and one virus, proceeding by the successive addition of one new species, first a bacteria, then a virus. Law and Morgan [8] refer to community assembly, one species at a time, as "community iteration", and we will follow the spirit of their ideas in this work. Table 3 describes the community iteration leading to the community of Table 1 . It is important that the sub-community at each stage of the iteration be permanent because it ensures that the intermediate communities are sufficiently stable so as to persist until a fortuitous mutational or colonization event allows further progression. Permanence is not a guarantee of long term persistence since environmental stochasticity may intervene to cause an extinction event, especially when a population is in a low part of its cycle. See Figure 1 below. However, our permanence result implies that should an extinction event occur, the resulting community is likely to be a permanent one and therefore recovery is possible.

We also show that time averages of species densities are asymptotic to appropriate equilibrium levels. Solutions of our chemostat-based model are highly oscillatory, apparently aperiodic, just as those observed for the LotkaVolterra system of Jover et al [7]. See Figure 1.

Perhaps it is interesting to note that the mathematical justification used to establish our results exploits the evolutionary successional sequence noted above by way of the principle of mathematical induction, establishing perma- 
Table 3 Assembly path for community in Table 1

\begin{tabular}{|c|c|c|}
\hline Iteration $\tau$ & Community members & Community with infection network \\
\hline 0 & $H_{1} ; V_{1}$ & $H_{1} \leftarrow V_{1}$ \\
\hline 1 & $H_{1}, H_{2} ; V_{1}$ & $H_{1} \leftarrow V_{1}, H_{2}$ \\
\hline 2 & $H_{1}, H_{2} ; V_{1}, V_{2}$ & $H_{1} \leftarrow V_{1}, V_{2}, H_{2} \leftarrow V_{2}$ \\
\hline 3 & $H_{1}, H_{2}, H_{3} ; V_{1}, V_{2}$ & $H_{1} \leftarrow V_{1}, V_{2}, H_{2} \leftarrow V_{2}, H_{3}$ \\
\hline 4 & $H_{1}, H_{2}, H_{3} ; V_{1}, V_{2}, V_{3}$ & $H_{1} \leftarrow V_{1}, V_{2}, V_{3}, H_{2} \leftarrow V_{2}, V_{3}, H_{3} \leftarrow V_{3}$ \\
\hline
\end{tabular}

nence in a given sub-community in the successional sequence by appealing to the permanence hypothesis of its predecessor in the sequence.

The competitive exclusion principle is critical to our approach. We will show that two virus strains cannot share the same set of bacterial hosts (i.e. cannot have the same host range) since one of the virus will be more efficient at exploiting the host and drive the other to extinction. Similarly, two bacterial strains cannot suffer infection by the same set of virus because the weaker competitor for nutrient will eventually be excluded. Therefore, the competitive exclusion principle drives the evolution of communities towards a nested infection structure.

As noted in [7], perfectly nested infection networks are generally only observed for very small host-virus communities. Because natural host-virus communities have strong tendency to be approximately nested in their infection structure, it is worth while to consider how the idealized nested network may have evolved. Mathematical modeling is especially useful for exploring these idealized scenarios. Furthermore, permanence, or persistence in mathematical models is known to be robust to model perturbations under appropriate conditions $[10,3,5]$ and therefore it should continue to hold for small deviations from a nested infection structure.

\section{A Chemostat-based Host-Virus Model}

The standard chemostat model of microbial competition for a single limiting nutrient [13] is modified by adding lytic virus. Our model is a special case of general host-virus models formulated in [2] which include viral latency. Let $R$ denote the nutrient which supports the growth of bacteria strains $H_{i}$; it is supplied at concentration $R_{0}$ from the feed. $V_{i}$ denote the various virus strains that parasitize the bacteria. Bacteria strain $H_{i}$ is characterized by its specific growth rate $f_{i}(R)$ and its yield $\gamma_{i}$. For simplicity, we assume that the yield is the same for all bacterial strains: $\gamma_{i}=\gamma$ is independent of $i$. At this point, we assume only that the specific growth rates $f_{i}$ are increasing functions of nutrient $R$, vanishing when $R=0$. Following [7], we assume that virus strain $V_{i}$ is characterized by its adsorption rate $\phi_{i}$ and its burst size $\beta_{i}$, both of which are assumed to be independent of which host strain it infects. $D$ denotes the dilution rate of the chemostat.

Let $I_{i}$ denote the host range of virus $V_{i}$ :

$$
I_{i}=\left\{j: V_{i} \text { infects host } H_{j}\right\},
$$


assumed to be nonempty for each $i$. Later, we will consider two special cases but for now we keep things general.

Our model is described by the following differential equations:

$$
\begin{aligned}
& R^{\prime}=D\left(R_{0}-R\right)-\sum_{j} \frac{1}{\gamma} f_{j}(R) H_{j} \\
& H_{i}^{\prime}=H_{i}\left(f_{i}(R)-D\right)-H_{i} \sum_{j: i \in I_{j}} \phi_{j} V_{j} \\
& V_{i}^{\prime}=\beta_{i} \phi_{i} V_{i} \sum_{j \in I_{i}} H_{j}-D V_{i}, 1 \leq i \leq n .
\end{aligned}
$$

Note that decay rates of virus and mortality of bacteria are ignored in (1). This is a crucial simplification for our results as it allows a reduction in dimension to be described below.

Non-dimensional quantities are identified below:

$N=\frac{R}{R_{0}}, B_{i}=\frac{H_{i}}{\gamma R_{0}}, D P_{i}=\phi_{i} V_{i}, \tau=D t, s_{i}=\frac{D}{\beta_{i} \phi_{i} \gamma R_{0}}, g_{i}(N)=\frac{f_{i}\left(R_{0} N\right)}{D}$.

Again using prime for derivative with respect to $\tau$, we have the equations

$$
\begin{aligned}
& N^{\prime}=1-N-\sum_{j} g_{j}(N) B_{j} \\
& B_{i}^{\prime}=B_{i}\left(g_{i}(N)-1\right)-B_{i} \sum_{j: i \in I_{j}} P_{j} \\
& P_{i}^{\prime}=s_{i}^{-1} P_{i}\left(\sum_{j \in I_{i}} B_{j}-s_{i}\right), 1 \leq i \leq n .
\end{aligned}
$$

Each virus strain is characterized by a single parameter $s_{i}$ which reflects its burst size $\beta_{i}$ and its adsorption rate $\phi_{i}$. Smaller $s_{i}$ translates to stronger ability at exploiting host.

Assume that the specific growth rate $g_{i}$ is a strictly increasing function of nutrient concentration and that there exists the break-even nutrient concentration $\lambda_{i}<1$ for strain $B_{i}$ defined by the balance of growth and dilution: $g_{i}\left(\lambda_{i}\right)=1$. We assume that the bacterial species are ordered such that

$$
0<\lambda_{1}<\lambda_{2}<\cdots<\lambda_{n}<1 \text {. }
$$

This implies that in the absence of virus, $B_{i}$ dominates $B_{j}$ if $i<j$ but that each bacteria is viable in the absence of the others. Indeed, classical chemostat theory $[13,16]$ implies that $B_{1}$ would eliminate all $B_{j}, j>1$ in the absence of the virus.

System (2) enjoys the usual chemostat conservation principle, namely that the total nutrient content of bacteria and virus plus free nutrient $T=N+$ $\sum_{i} B_{i}+\sum_{i} s_{i} P_{i}$ must come into balance with the input of nutrient: $T^{\prime}=1-T$. On the exponentially attracting invariant set $T=1$ we can drop the equation 
for $N$ from (2) and replace $N$ by $1-\sum_{i} B_{i}-\sum_{i} s_{i} P_{i}$. We observe that this reduction in dimension afforded by the conservation principle, crucial for our analysis, is lost if viral decay and/or bacterial death rates are included in the model.

As a final model simplification, linear specific growth rates for bacteria

$$
g_{i}(N)=r_{i} N
$$

are assumed where, by (3), we must have

$$
1<r_{n}<r_{n-1}<\cdots<r_{2}<r_{1} .
$$

Then $\lambda_{i}=1 / r_{i}$. The result is the system with Lotka-Volterra structure

$$
\begin{aligned}
& B_{i}^{\prime}=r_{i} B_{i}\left(1-\frac{1}{r_{i}}-\sum_{j}\left(B_{j}+s_{j} P_{j}\right)\right)-B_{i} \sum_{j: i \in I_{j}} P_{j} \\
& P_{i}^{\prime}=s_{i}^{-1} P_{i}\left(\sum_{j \in I_{i}} B_{j}-s_{i}\right), 1 \leq i \leq n .
\end{aligned}
$$

In the absence of virus and in view of $(3)$, there are only single-population bacterial equilibria for $(5)$. Let $E_{i}=\left(1-\lambda_{i}\right) e_{i}$ denote the equilibrium where only host strain $B_{i}$ is present. Here, $e_{i}$ is the unit vector with all components zero except the $i$ th which is one. In the absence of virus, $E_{1}$ attracts all solutions with $B_{1}(0)>0[13,16]$.

We consider two special cases for the bipartite infection network in this paper. The nested infection network (NIM), so named by Jover et al [7], is characterized by $I_{i}=\{j: j \leq i\}$. Virus strain $V_{i}$ infects all host strains $H_{j}$ for $j \leq i$. Thus, strain $V_{1}$ specializes on host $H_{1}$ while strain $V_{n}$ is a generalist, infecting all host strains. As $i$ increases, virus strain $V_{i}$ becomes more generalist, less of a specialist; the index $i$ is indicative of the number of host strains $V_{i}$ infects. In particular, the superiority rank of a bacterial strain is inversely related to the number of virus strains that infect it. Strain $B_{1}$ is the best competitor in virus-free competition for nutrient but it can be infected by all the virus strains, while strain $B_{n}$ is the worst competitor for nutrient but can be infected only by virus strain $P_{n}$.

Also considered is the monogamous infection network (MIN) $I_{i}=\{i\}$ where there is a one-to-one relation between virus and host. In this case, virus are highly selective of whom they may infect. This model was considered by Thingstad [15].

\section{Nested Infection Network}

The model equations in this case are:

$$
B_{i}^{\prime}=r_{i} B_{i}\left(1-\frac{1}{r_{i}}-\sum_{j}\left(B_{j}+s_{j} P_{j}\right)\right)-B_{i} \sum_{j \geq i} P_{j}
$$




$$
P_{i}^{\prime}=s_{i}^{-1} P_{i}\left(\sum_{j \leq i} B_{j}-s_{i}\right) \quad 1 \leq i \leq n .
$$

Next we consider equilibria where all or nearly all host and virus are present. Only the equilibria which play a role in our results will be mentioned. if

There exists an equilibrium $E^{*}$ with $B_{i}$ and $P_{i}$ positive for all $i$ if and only

$$
s_{1}<s_{2}<s_{3}<\cdots<s_{n}
$$

and

$$
\frac{r_{n}}{1+Q_{n}}>1
$$

hold, where $Q_{1}=r_{1} s_{1}$ and $Q_{n}=\sum_{i=1}^{n} s_{i}\left(r_{i}-r_{i+1}\right), n>1$, where $r_{n+1}=0$. In fact,

$$
\begin{aligned}
& B_{1}^{*}=s_{1}, B_{j}^{*}=s_{j}-s_{j-1}, j>1, \\
& P_{j}^{*}=\frac{r_{j}-r_{j+1}}{1+Q_{n}}, j<n, P_{n}^{*}=\frac{r_{n}}{1+Q_{n}}-1 .
\end{aligned}
$$

The positive equilibrium $E^{*}$ is unique. On rearranging the sum, we find that $Q_{n}=\sum_{i=1}^{n} r_{i} B_{i}^{*}$.

(7) and (8) also imply the existence of a unique equilibrium $E^{\dagger}$ with all components positive except for $P_{n}=0$. In fact,

$$
\begin{aligned}
& B_{j}^{\dagger}=B_{j}^{*}, 1 \leq j<n, \\
& B_{n}^{\dagger}=B_{n}^{*}+\left(1-\frac{1+Q_{n}}{r_{n}}\right), \\
& P_{j}^{\dagger}=P_{j}^{*}\left(\frac{1+Q_{n}}{r_{n}}\right), j<n, P_{n}^{\dagger}=0 .
\end{aligned}
$$

As the existence of a positive equilibrium is a necessary condition for permanence, it is instructive to elaborate on the implications of the result above. We may obviously view the inequalities (4) as simply a convenient way to order the microbes but then we must explain the inequalities (7) as they are necessary for the existence of a positive equilibrium and therefore, for permanence of the bacteria-virus community with nested infection network. Since the $s_{i}$ are inversely related to virus efficiency at exploiting host, (7) requires that virus that are efficient at exploiting bacteria have smaller host range. In addition, inequality (8) must be satisfied. In terms of the unscaled parameters it becomes

$$
\frac{r_{1}-r_{2}}{\phi_{1} \beta_{1}}+\frac{r_{2}-r_{3}}{\phi_{2} \beta_{2}}+\cdots+\frac{r_{n-1}-r_{n}}{\phi_{n-1} \beta_{n-1}}+\frac{r_{n}}{\phi_{n} \beta_{n}}<\left(r_{n}-1\right) \frac{\gamma R_{0}}{D},
$$

This may be viewed as a restriction in many different ways as it involves both biological and physical parameters. For example, it holds automatically if sufficient nutrient is provided to the chemostat or if the flow rate is sufficiently low. 
Otherwise, it may be viewed as a restriction on the size $2 n$ of the community, or on the efficiency of the virus $\beta_{i} \phi_{i}$.

Notice that $Q_{n}=Q_{n-1}+r_{n} B_{n}^{*}$ which together with (4) implies that $\frac{r_{k}}{1+Q_{k}}>\frac{r_{n}}{1+Q_{n}}$ for $1 \leq k<n$. Therefore, (7) and (8) imply the existence of a unique family of equilibria $E_{k}^{*}$ with $B_{j}, P_{j}=0, j>k$ described by (9) but with $Q_{k}$ replacing $Q_{n}$. Another family of equilibria, $E_{k}^{\dagger}$, exists with $B_{j}=0, j>k$ and $P_{j}=0, j \geq k$ described by (10) but with $Q_{k}$ replacing $Q_{n}$.

Not surprisingly, the density of $B_{i}$ at the positive equilibrium $E^{*}$ is less then the density of $B_{i}$ at its equilibrium $E_{i}$. Also we note that $P_{j}^{\dagger}<P_{j}^{*}$, $B_{j}^{\dagger}=B_{j}^{*}$ for $j<n$ and $B_{n}^{\dagger}>B_{n}^{*}$.

Free nutrient levels at $E^{*}$ and $E^{\dagger}$ are revealing. At $E^{\dagger}$, the (scaled) free nutrient level is given by $\lambda_{n}=1 / r_{n}$, the same as at $E_{n}$ where only bacteria strain $B_{n}$ is present with no virus. At $E^{*}$, the nutrient level is greater than at $E^{\dagger}$. It is given by $\frac{1}{1+Q_{n}}$, thus the ratio of the nutrient levels is precisely (8). Chao et al [2] refer to $E^{*}$ as a "phage-limited" community while $E^{\dagger}$ is referred to as a "nutrient-limited" one when $k=1$.

The inequality (8) implies that $E^{\dagger}$ is unstable to invasion by $P_{n}$ since

$$
\left.\frac{s_{n} P_{n}^{\prime}}{P_{n}}\right|_{E^{\dagger}}=\left(1-\frac{1+Q_{n}}{r_{n}}\right)>0 .
$$

Computer simulations of (6) provide additional insight. We adopted parameter values from Table 2 of Bohannan and Lenski [1] from which we computed $r_{i}$ and $s_{j}$ using our scaled variables. The Monod growth rates for bacteria used in [1] were linearized to obtain our $r_{i}$; for example, $r_{1}=\frac{\psi_{A} R_{0}}{K_{A} D}=26.64$. For parameter $r_{2}$, we replaced $\psi_{A}=0.7726 h^{-1}$ by $\psi_{B}=0.7027 h^{-1}$ but we retained $K_{A}=0.0727 \mu \mathrm{g} \mathrm{ml}^{-1}$ for the half saturation constant. As for $r_{3}$, since there is no counterpart in [1], we replaced $\psi_{A}$ by $0.6326 h^{-1}$ to maintain an equal decrement as from $\psi_{A}$ to $\psi_{B}$, and we retained $K_{A}$. We used $\beta_{i}=100$ for all $i$ and chose adsorption rates $\phi_{1}=2 \times 10^{-7} \mathrm{ml} \mathrm{h}^{-1}, \phi_{2}=1 \times 10^{-7} \mathrm{ml} \mathrm{h}^{-1}$ as in [1] and $\phi_{3}=0.5 \times 10^{-7} \mathrm{mlh}^{-1}$ was taken to maintain an equal decrement. The results appear in Table 4

Table 4 Parameter values used in simulations

\begin{tabular}{|c|c|}
\hline Parameter & value \\
\hline \hline$r_{1}$ & 26.6 \\
\hline$r_{2}$ & 24.2 \\
\hline$r_{3}$ & 21.8 \\
\hline$s_{1}$ & 0.04 \\
\hline$s_{2}$ & 0.08 \\
\hline$s_{3}$ & 0.16 \\
\hline
\end{tabular}

Figure 1 provides illuminating simulations of (6) for the case $n=3$. All simulations were performed in MATLAB using solver ode45. See Table 4 for a list of parameter values. Initial data, if nonzero, are taken to be $B_{1}(0)=$ 
$s_{1}, B_{i}(0)=s_{i}-s_{i-1}, i>1$, and $P_{i}(0)=0.1$. For the top row, all initial population densities are nonzero. Observe that free nutrient level is high in this case because $P_{3}$, the dominant virus, keeps $B_{3}$ at low density. The bacterial community is "phage limited" in this case. In the second row, initial data are positive except $P_{3}(0)=0$. Observe that free nutrient levels are much lower than for the top row because $B_{3}$ is free to consume it. The bacterial community is "nutrient limited" in this case. In the third row has initial data are positive except for $B_{3}(0)=P_{3}(0)=0$.

Viewing Figure 1 from bottom to top as a successional sequence, we see that it is driven by a sequence of opportunities. In the bottom row hostphage community, nutrient is plentiful providing an opportunity for a suitable bacterial invasion. In the middle row, the invading bacteria $B_{2}$ is at high levels providing and opportunity for a suitable virus.

Figure 2 depicts the invasion of the $B_{1}-P_{1}$ equilibrium by $B_{2}$ followed by the introduction of $P_{2} . B_{2}$ is introduced at $T=20$ at the level $B_{1}(T=$ 20)/100 mimicking a mutational event; similarly, $P 2$ is introduced at $T=40$ in the concentration $P_{1}(T=40) / 100$. Each time unit is equivalent to 5 hours. Note the sharp exponential decline of $P_{1}$ following the introduction of $B_{2}$ and the rapid decline of $B_{2}$ following the introduction of $P_{2}$. The extent of these declines is remarkable.

Fig. 1 Nested Infection Network with $n=3$. Top: positive initial data; Middle: $P_{3}=0$; Bottom: $P_{3}=B_{3}=0$.
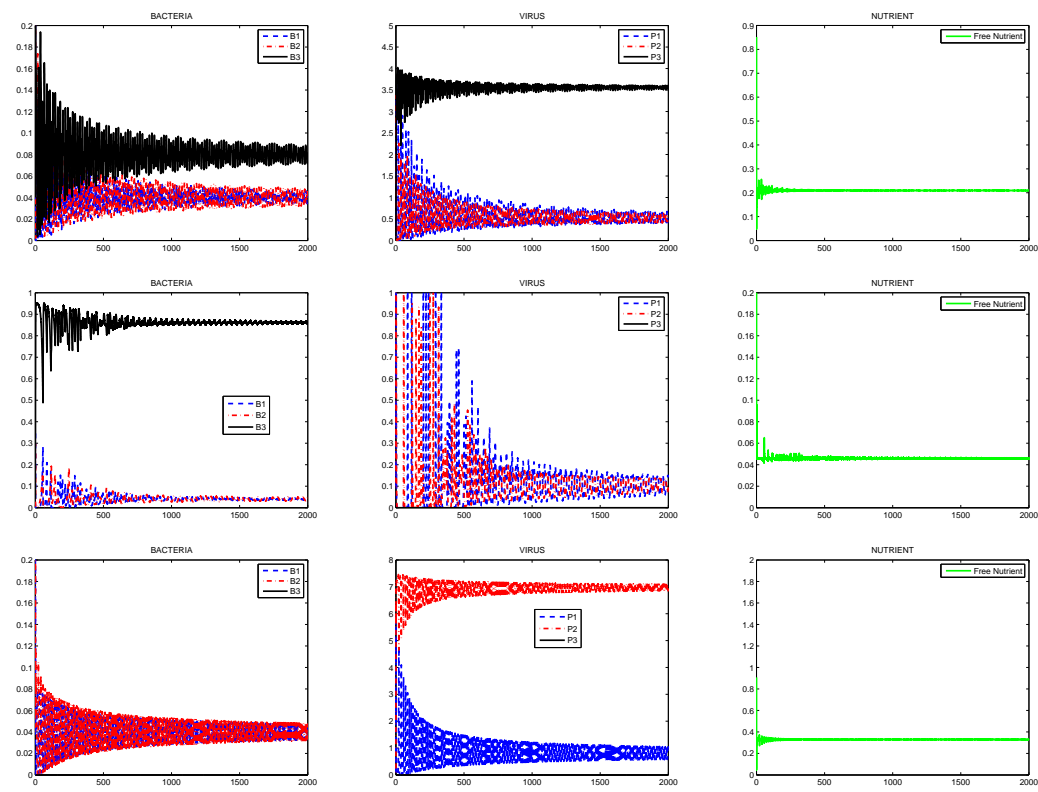
Fig. $2 B 2$ invades $B 1-P 1$ equilibrium at $T=20$ (left). Subsequently, $P 2$ invades at $T=40$ (right).
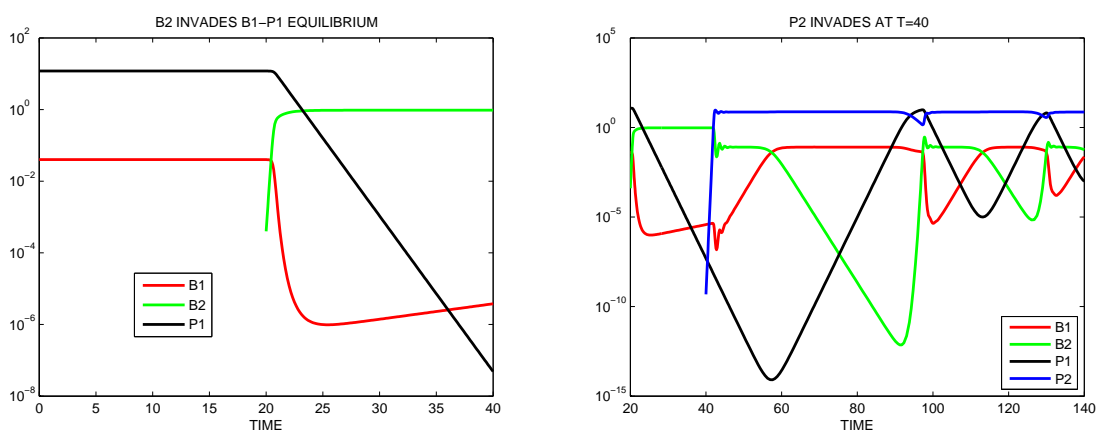


\section{Monogamous Infection Network}

The model equations in this case are:

$$
\begin{aligned}
& B_{i}^{\prime}=r_{i} B_{i}\left(1-\frac{1}{r_{i}}-\sum_{j}\left(B_{j}+s_{j} P_{j}\right)\right)-B_{i} P_{i} \\
& P_{i}^{\prime}=s_{i}^{-1} P_{i}\left(B_{i}-s_{i}\right) \quad 1 \leq i \leq n .
\end{aligned}
$$

The principal equilibria for a monogamous infection network are now described. There exists an equilibrium $E^{*}$ with $B_{i}$ and $P_{i}$ positive for all $i$ if and only if

$$
\frac{r_{n}}{1+\tilde{Q}_{n}}>1, \quad \tilde{Q}_{n}=\sum_{i} r_{i} s_{i}
$$

In fact,

$$
\begin{aligned}
B_{j}^{*} & =s_{j}, j \geq 1, \\
P_{j}^{*} & =\frac{r_{j}}{1+\tilde{Q}_{n}}-1, j \geq 1 .
\end{aligned}
$$

The positive equilibrium $E^{*}$ is unique.

(13) also implies the existence of a unique equilibrium $E^{\dagger}$ with all components positive except for $P_{n}=0$. In fact,

$$
\begin{aligned}
B_{j}^{\dagger} & =B_{j}^{*}, 1 \leq j<n, \\
r_{n} B_{n}^{\dagger} & =r_{n}-\left(1+\sum_{i<n} s_{i} r_{i}\right), \\
P_{j}^{\dagger} & =\frac{r_{j}}{r_{n}}-1, j<n, P_{n}^{\dagger}=0 .
\end{aligned}
$$

In contrast to the case of NIN, the existence of a positive equilibrium in case MIN, a necessary condition for permanence, does not place order restrictions on the $s_{i}$ as in (7). However, the requirement that (13) holds, together with (4), implies that $0<s_{i}<1$ for all $i$. Inequality (13) is a stronger restriction than (8), as can be seen by comparing (11) with its counterpart for MIN:

$$
\frac{r_{1}}{\phi_{1} \beta_{1}}+\frac{r_{2}}{\phi_{2} \beta_{2}}+\cdots+\frac{r_{n-1}}{\phi_{n-1} \beta_{n-1}}+\frac{r_{n}}{\phi_{n} \beta_{n}}<\left(r_{n}-1\right) \frac{\gamma R_{0}}{D}
$$

We may conclude that given $n$ bacterial strains and $n$ virus strains, more nutrient $R_{0}$ must be supplied to the chemostat to support an MIN community than to support a NIN community, though in case of NIN (7) must be satisfied.

Equilibrium $E^{\dagger}$ is unstable to invasion of $P_{n}$ if (13) holds since

$$
\left.\frac{r_{n} s_{n} P_{n}^{\prime}}{P_{n}}\right|_{E^{\dagger}}=r_{n} B_{n}^{\dagger}-r_{n} s_{n}=r_{n}-\left(1+\tilde{Q}_{n}\right)>0
$$

In the same way as for the case NIN, (14) implies the existence of a unique family of equilibria $E_{k}^{*}$ and $E_{k}^{\dagger} . E_{k}^{*}$ is characterized by $B_{i}>0$ and $P_{i}>0$ if 
and only if $i \leq k$ while $E_{k}^{\dagger}$ is as for $E_{k}^{*}$ except $B_{i}>0$ if and only if $i<k$. We have no need for a complete list of equilibria.

Free nutrient levels at $E^{*}$ and $E^{\dagger}$ have the same relation as for the case NIN. At $E^{\dagger}$, the (scaled) free nutrient level is given by $\lambda_{n}=1 / r_{n}$, the same as at $E_{n}$ where only bacteria strain $B_{n}$ is present with no virus. At $E^{*}$, the nutrient level is given by $\frac{1}{1+\tilde{Q}_{n}}$, thus the ratio of the nutrient level at $E^{*}$ to that at $E^{\dagger}$ is precisely (13).

Figure 3 provides illuminating simulations of (12) for the case $n=3$. See Table 4 for a list of parameter values. Initial data, if nonzero, are taken to be $B_{i}(0)=s_{i}$ and $P_{i}(0)=0.1$. For the top row, all initial population densities are nonzero. As in Figure 1 for the case NIN, free nutrient level is high in this case because $P_{3}$, the dominant virus, keeps $B_{3}$ at low density. The bacterial community is "phage limited" in this case. In the second row, initial data are positive except $P_{3}(0)=0$. Observe that free nutrient levels are slightly lower than for the top row because $B_{3}$ is free to consume it. In the third row, the initial data satisfies $B_{3}(0)=P_{3}(0)=0$, the others being positive.

Fig. 3 Monogamous Infection Network with $n=3$. Top: positive initial data; Middle: $P_{3}=0 ;$ Bottom: $P_{3}=B_{3}=0$
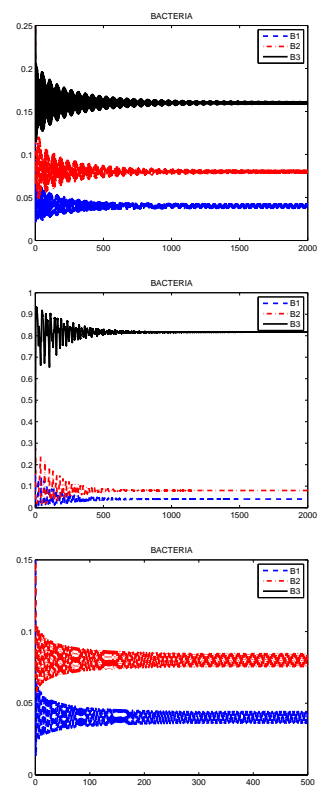
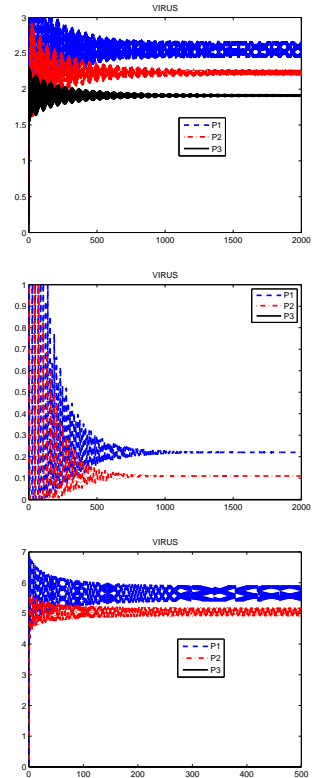
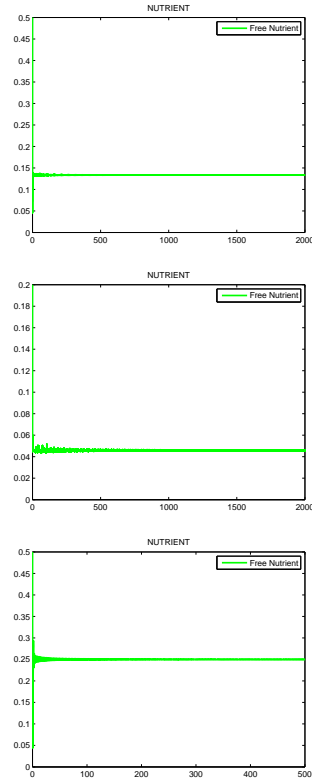


\section{Permanence for Nested and Monogamous Networks}

Our main result follows. It applies to both cases. We use the notation $(\mathbf{B}, \mathbf{P})=$ $\left(B_{1}, B_{2}, \cdots, B_{n}, P_{1}, \cdots, P_{n}\right)$ for a solution of (6) or of (12). A detailed proof is given in Supplementary Materials.

Theorem 1 Let $1 \leq k \leq n$. Assume (7) and (8) in case of NIN and assume (13) in case MIN.

(a) There exists $\epsilon_{k}>0$ such that if $(\mathbf{B}, \mathbf{P})$ is a solution satisfying

$$
B_{i}(0)>0,1 \leq i \leq k, \quad P_{j}(0)>0,1 \leq j \leq k-1,
$$

then there exists $T>0$ such that for all $t>T$ :

$$
B_{i}(t) \geq \epsilon_{k}, 1 \leq i \leq k \text { and } P_{j}(t) \geq \epsilon_{k}, 1 \leq j \leq k-1 .
$$

(b) There exists $\epsilon_{k}>0$ such that if $(\mathbf{B}, \mathbf{P})$ is a solution satisfying

$$
B_{i}(0)>0, P_{i}(0)>0,1 \leq i \leq k,
$$

then there exists $T>0$ such that for all $t>T$ :

$$
B_{i}(t) \geq \epsilon_{k}, P_{i}(t) \geq \epsilon_{k}, 1 \leq i \leq k .
$$

Observe that in both cases (a) and (b) we require only certain prescribed components of the initial data to be positive but we make no restrictions on the other components except, of course, that they are nonnegative. This means that a community consisting of only a single virus population and a single bacteria population that is susceptible to infection by the virus is permanent. Furthermore, the addition of a new bacterial population, say through a mutation or a colonization event, results in a permanent community as well provided that the newcomer is resistant to infection. And the subsequent addition of a new virus population, capable of infecting the new bacteria and perhaps the original one as well, results in a permanent community, and so on.

Theorem 5.2.3 in [6] together with the previous result implies for every solution of (6) or of (12) starting with all components positive, we have that

$$
\frac{1}{t} \int_{0}^{t} B_{i}(s) d s \rightarrow B_{i}^{*}, \frac{1}{t} \int_{0}^{t} P_{i}(s) d s \rightarrow P_{i}^{*}
$$

where $B_{i}^{*}, P_{i}^{*}$ are as in $(9)$ or $(14)$.

For every solution of (6) or of (12) starting with all components positive except $P_{n}(0)=0$, we have that

$$
\frac{1}{t} \int_{0}^{t} B_{i}(s) d s \rightarrow B_{i}^{\dagger}, \frac{1}{t} \int_{0}^{t} P_{i}(s) d s \rightarrow P_{i}^{\dagger}
$$

where $B_{i}^{\dagger}, P_{i}^{\dagger}$ are as in (10) or (15). 


\section{Discussion}

We have shown that a community consisting of an equal number of bacteria and bacteriophage populations in a chemostat in which bacteria compete for a single nutrient and for which the infection network is perfectly nested is permanent provided that (1) the bacteria that are superior competitors for nutrient devote the least effort to defence against infection, (2) the virus that are the most efficient at infecting host have the smallest host range, and (3) sufficient nutrient is input to the chemostat. Jover et al [7] raised the issue of whether nested infection networks with the tradeoffs described above result in permanence for their comparable Lotka-Volterra model.

The same permanence result is shown to hold for a monogamous infection network without the tradeoffs (1) and (2). However, a larger community size $2 n$, namely $n$ bacterial strains and $n$ virus strains, can be supported for a given nutrient concentration in the feed to the chemostat if the infection network is nested than if it is monogamous.

For both types of infection networks $I=M I N$ or $I=N I N$, we show that starting with a single bacterial population, a community consisting of $n$ bacterial populations and $n$ (or $n-1$ ) virus populations with infection network of type $I$ can arise through a succession of permanent sub-communities, each with infection network of type $I$, by the successive addition of one new population, first a virus, then a new bacteria, alternating in this fashion. This is important because it ensures that the intermediate communities are sufficiently stable so as to persist until a fortuitous mutational or colonization event allows further progression. Permanence is not a guarantee of long term persistence since environmental stochasticity may intervene to cause an extinction event, especially when a population is at a low part of its cycle. See Figure 1 and Figure 2. However, our permanence result implies that should an extinction event occur, the resulting community is likely to be a permanent one and therefore recovery is possible.

As noted by Bohannan and Lenski [1], a successional sequence arises in the setting of a chemostat, starting with a single bacteria population, provided sufficient nutrient is input to the chemostat. The bacteria are capable of converting much of the nutrient to bacterial biomass providing an opportunity for a virus. Once a host-virus community is established, nutrient levels rise providing a niche for another bacterial population, even one which is an inferior competitor for nutrient provided it is resistant to infection. If it is fully resistant to infection, the new bacteria population can dominate the original one so now there is an opportunity for a mutant (or rare variant) virus that can infect the new host.

Of course, natural communities of bacteria and virus will have complicated infection networks that are not perfectly nested or monogamous. Jover et al [7] show that observed infection networks have a tendency to be approximately nested. Furthermore, the assumptions made in our mathematical model, the same ones made in [7], are quite special. For example, it was assumed that virus adsorption rates and burst sizes are independent of the host bacteria 
and that yield constants are identical for all bacterial populations. Specific growth rates of bacteria are assumed to be linear in nutrient concentration and bacterial mortality is ignored. Virus latency and viral decay has been ignored in our model. Therefore, our results for these idealized special cases can at best suggest that large communities of bacteria and virus with complicated infection networks can evolve by the sequential addition of new populations.

Acknowledgements Both authors were partially supported by National Science Foundation grant DMS-0918440.

\section{References}

1. Bohannan BJM and Lenski RE (2000) Linking genetic change to community evolution: insights from studies of bacteria and bacteriophages, Ecology Letters 3: 362-377.

2. Chao L, Levin BR, Stewart FM (1977) A Complex Community in a Simple Habitat: An Experimental Study with Bacteria and Phage, Ecology 58: 369-378.

3. Garay B and Hofbauer J (2003) Robust Permanence for ecological differential equations, minimax, and discretizations, SIAM J. Math. Anal. 34: 1007-1039.

4. Han Z and Smith HL (2012) Bacteriophage-Resistant and Bacteriophage-Sensitive Bacteria in a Chemostat, Math. Biosciences and Eng. 9: 737-765.

5. Hirsch MW, Smith HL, Zhao XQ (2001) Chain transitivity, attractivity and strong repellors for semidynamical systems, J.Dynamics and Diff. Eqns. 13:107-131.

6. Hofbauer J and Sigmund K (1998) Evolutionary games and Population Dynamics, Cambridge Univ. Press.

7. Jover LF, Cortez MH, Weitz JS (2013) Mechanisms of multi-strain coexistence in hostphage systems with nested infection networks, Journal of Theoretical Biology 332: 65-77

8. Law R and Morton RD (1996) Permanence and the assembly of ecological communities, Ecology 77: 762-775.

9. May RM and Leonard WJ (1975) Nonlinear aspects of competition between three species, SIAM J. Applied Math. 29: 243-253.

10. Schreiber S (2000) Criteria for $C^{r}$ robust permanence, J. Diff. Eqns. 162: 400-426.

11. Smith HL and Thieme HR (2011) Dynamical Systems and Population Persistence, GSM 118, Amer. Math. Soc.

12. Smith HL and Thieme HR (2012) Persistence of Bacteria and Phages in a Chemostat, Journal of Mathematical Biology 64: 951-979.

13. Smith HL and Waltman P (1995) The Theory of the Chemostat, Cambridge Univ. Press.

14. Thieme H (1993) Persistence under relaxed point-dissipativity (with applications to an endemic model), SIAM J. Math. Anal. 24: 407-435.

15. Thingstad TF (2000) Elements of a theory for the mechanisms controlling abundance, diversity, and biogeochemical role of lytic bacterial viruses in aquatic systems, Limnol. Oceanogr. 45: 1320-1328.

16. Tilman D (1982) Resource Competition and Community Structure, Princeton Univ. Press.

17. Weitz JS, Hartman H, Levin SA (2005) Coevolutionary arms races between bacteria and bacteriophage, PNAS 102:9535-9540. 